Wilfried Schütte/Thomas Spranz-Fogasy

\title{
DIDAKTISCHE MÖGLICHKEITEN DES VIDEOEINSATZES IN HYPERMEDIALEN SYSTEMEN DER GESPRÄCHSANALYSE
}

\begin{abstract}
Hypermedia und Internet revolutionieren die Gesellschaft in vielen Lebens- und Arbeitsbereichen. Sie sind auch in der Linguistik nicht nur zum Forschungsgegenstand, sondern auch zum Präsentationsmedium geworden. Auch und besonders in der Gesprächsanalyse werden die Möglichkeiten der Darstellung und der Analyse durch den Einsatz elektronischer Systeme um ein Vielfaches erweitert. In diesem Beitrag möchten wir darüber informieren, wie und zu welchem Zweck Videodateien in verschiedenen online oder offline verfügbaren Systemen zur Gesprächsforschung eingesetzt werden, und versuchen, den Stellenwert dieses Einsatzes im gesprächsanalytischen Arbeitsprozess aufzuzeigen. Nach einer Darstellung der bisherigen wesentlichen Entwicklungsschritte des Gesprächsanalytischen Informationssystems GAIS und einem Überblick über andere Hypermediasysteme zeigen wir, wie man mit Hilfe von Videos die Flüchtigkeit des originalen Kommunikationsereignisses nachbilden oder in spezifischer Weise auf dieses Ereignis zugreifen kann. Ferner zeigen wir, wie sich der Videoeinsatz mit Phasen des gesprächsanalytischen Arbeitsprozesses verknüpfen lässt, und plädieren für eine Integration der didaktischen Vermittlung in diesen Prozess. Wir beschäftigen uns hier also mit den didaktischen Einsatzmöglichkeiten für Lehrende und den Lernmöglichkeiten für Studierende, die hypermediale Systeme in der Gesprächsanalyse bieten.
\end{abstract}

Hypermedia and the internet are revolutionising society in many areas of life and work. In linguistics, too, they have become not only the object of research, but also the medium of presentation. This is especially the case in conversation analysis, where the use of electronic systems has greatly extended the possibilities of presentation and analysis. In this article we would like to report on how and for what purposes video files are used for conversation research in various on-line or off-line systems, and to try to show the value of these tools in work on conversation analysis. We begin with an outline of the most important steps so far in the development of the Information System for Conversation Analysis (GAIS) and an overview of other hypermedia systems, and go on to show how videos can be used to imitate the fleeting nature of the original communication event or to access this event in a specific way. Then we show how the use of video can be linked with phases of conversation analysis and argue for an integration of teaching into this process. We are thus concerned here with the didactic applications for teachers and the learning opportunities for students which hypermedia systems can offer in conversation analysis.

\section{Ausgangspunkte}

Seit 2000 haben Werner Kallmeyer und Wilfried Schütte Material für gesprächsanalytische Seminare an der Universität Mannheim auf CD-ROM und online zusammengestellt. Dieses Material umfasste Transkripte und digitalisierte Videoaufnahmen zu Diskursausschnitten aus den IDS-Gesprächskorpora; es konnte für Übungsaufgaben, für eine sitzungsübergreifende Gruppenarbeit, für analytische Demonstrationen der Dozenten und für Hausarbeiten der Studierenden eingesetzt werden. Zudem sahen wir es als sinnvoll an, gesprächsanalytische Arbeiten, die wir als Dozenten in den Seminaren vorgestellt und diskutiert haben oder die im Rahmen von Seminararbeiten der Studierenden entstanden, den SeminarteilnehmerInnen und darüber hinaus der wissenschaftlichen Öffentlichkeit in digitaler Form und online zur Verfügung zu stellen. 
Den Anstoß zu einer systematischeren Entwicklung des Gesprächsanalytischen Informationssystems GAIS gab dann die Beteiligung am Projektverbund „PortaLingua“, dem „Hochschulverbund zum Einsatz curricularer Bausteine in der Sprach- und Kommunikationswissenschaft - multimediale Lehr- und Lernressourcen für Präsenzlehre, Selbststudium und Weiterbildung“. ${ }^{1}$ In dieser Entwicklungsphase wurde GAIS als multimediales linguistisches Lern- und Informationssystem konzipiert, das Grundlagenwissen zur Gesprächsanalyse anbieten und in der universitären Lehre eingesetzt werden sollte, um das Studium zu flexibilisieren und effektiver zu machen. Der Nutzen für die Studierenden ergab sich vor allem aus einer Verbindung von theoretischen Darstellungen mit exemplarischen Sequenzanalysen, die zum Beleg der analytischen Aussagen mit Videos, Tönen, Transkripten und prosodischen Analysen synoptisch angereichert oder verlinkt waren.

Um das System für Autoren innerhalb und außerhalb des IDS nutzbar zu machen, wurde es 2004-2005 auf ein Content-Management-System mit neuem Layout und neuer Benutzerverwaltung umgestellt. Benutzer können sich darin in verschiedenen Benutzergruppen registrieren lassen, damit verbunden sind dann unterschiedliche Benutzerrechte der Rezeption, Inhaltsverwaltung oder Produktion von GAIS-Inhalten. ${ }^{2}$

$\mathrm{Zu}$ den Möglichkeiten von GAIS gehört auch die Einstellung und Wiedergabe von Videos, die mittlerweile als Datengrundlage zum festen Bestand der Gesprächsanalyse gehören. Im Folgenden möchten wir verschiedene Hypermedia-Systeme zur Gesprächsanalyse kurz vorstellen ${ }^{3}$ und sie unter dem Aspekt vergleichen, wie sie mit Videos umgehen. Geht man davon aus, dass der Einsatz und die Präsentation von Videos didaktischen Zielsetzungen folgen muss, stellen sich zwei Grundfragen: Was soll die Rezeption des Videos dem Systemnutzer vermitteln? Nimmt das System die mit dem Video verbundenen Möglichkeiten einer unmittelbaren sinnlichen Anschauung des nonverbalen Verhaltens der Gesprächsbeteiligten im analytischen Begleittext angemessen wahr?

\section{Hypermedia-Systeme zur Gesprächsanalyse}

Neben GAIS werden mittlerweile eine ganze Reihe von Hypermedia-Systemen zur Gesprächsforschung online und offline angeboten. Es handelt sich dabei teilweise um didaktische Angebote für Lehre und Selbststudium in verschiedenen Informations- und Schwierigkeitsgraden sowie um Informationsangebote und Online-Publikationsmög-

\footnotetext{
${ }^{1}$ Konsortialführer für diesen Projektverbund war die Universität Essen; er wurde vom Bundesministerium für Bildung und Forschung (BMBF) im Rahmen des Förderprogramms „NMB - Neue Medien in der Bildung“ gefördert. „PortaLingua“ wurde darin zu einem WWW-Portal für Sprach- und Kommunikationswissenschaften ausgebaut (vgl. http://www.portalingua.uni-essen.de/). Das IDS war darin mit den zwei Teilprojekten GAIS und ProGr@mm (http://www.ids-mannheim.de/gra/progr@mm.html) beteiligt. Die Ergebnisse des Projekts werden dargestellt in Heidtmann (2004), Heidtmann/Schütte/Steiger (2003) und Schütte (2004). Stand aller Screenshots von Online-Angeboten und aller URLs in diesem Beitrag ist der April 2006.

${ }^{2}$ GAIS wurde im neuen System Ende 2005 unter der Adresse http://gais.ids-mannheim.de freigeschaltet.

${ }^{3}$ Konzeption und Inhalte der hier behandelten und weiterer multimedialer Informations- und Lehrsysteme werden in Cölfen/Schütte/Spranz-Fogasy (2006) beschrieben.
} 
lichkeiten für Interessierte und Experten. Die wesentlichen derzeit bekannten Informationssysteme sind: ${ }^{4}$

- EGon - Einführung in die Gesprächsforschung online, TU Chemnitz, Werner Holly/Michael Klemm (http://www.tu-chemnitz.de/phil/gf/)

- eHype-Entwicklung hypermedialer Onlinekomponenten zur Vermittlung von Ergebnissen der Gesprächs- und Interaktionsanalyse zwischenmenschlicher Kommunikation, Fachhochschule Fulda, Werner Nothdurft (http://www.fh-fulda.de/index.php?id=1051)

- Informationsportal Gesprächsforschung - Institut für Gesprächsforschung, Martin Hartung (http://www.gespraechsforschung.de $)^{5}$

- Miteinander Reden - Ein Projekt zur Erforschung dialogischer HypermediaProduktion, Universität Essen, Hermann Cölfen (http://www.sprachmuseum.de oder http://www.linse.uni-essen.de/miteinanderreden/index.htm)

- Studien-CD Linguistik-Multimediale Einführungen und interaktive Übungen zur germanistischen Sprachwissenschaft (von Christa Stocker u.a.), Max Niemeyer Verlag Tübingen und Universität Zürich 2004.

Die meisten dieser Systeme werden online angeboten, allerdings z.T. (eHype und EGon) nur für registrierte Nutzer. Eine Ausnahme bildet hier die Studien-CD Linguistik als CDROM, die in Macromedia-Flash programmiert wurde und als kommerzielles Produkt an ein Buch und damit an einen Verlag mit dessen Vertriebskanälen gebunden ist. ${ }^{6}$ Eine Begründung für die Wahl des Online-Angebots gibt es beispielsweise bei Miteinander Reden indirekt über die Zielsetzung der Entwicklung eines Online-,,Lernpäckchens“: Während der Entwicklungsphase sollte das „Lernpäckchen“ von rund 40 SchülerInnen und Studierenden - sozusagen stellvertretend für die Gruppe der späteren Nutzer - und einer Expertengruppe fortlaufend und regelmäßig kommentiert, kritisiert und in Teilen weiterentwickelt werden. Diese Form der im Dialog ausgehandelten gemeinsamen Entwicklung eines Hypermedia-Produkts nennt der Autor Hermann Cölfen dialogische Hypermedia-Produktion (Cölfen 2006).

Ein hypermediales System online anzubieten, hat mehrere Vorteile:

- In der Produzentenperspektive: Das System ist fortwährend aktualisierbar.

- In der Rezipientenperspektive: Die Nutzung ist personalisierbar - registrierte und angemeldete Nutzer können u.a. Lesezeichen setzen, ihre Lernfortschritte im System dokumentieren, Suchmuster speichern oder eigene Inhalte eingeben.

\footnotetext{
${ }^{4}$ Ein Angebot aus teachsam - Lehren und Lernen online im Fachbereich Deutsch $\rightarrow$ Arbeitsbereich Linguistik $\rightarrow$ Gesprächsanalyse soll unberücksichtigt bleiben, weil es sich dabei um ein reines Textangebot handelt, ohne hypermediale Aufbereitung, d.h. ohne Beispiele in Form von Audio-, Videodateien und Transkripten (s. http://www.teachsam.de/deutsch/d_lingu/gespraechsanalyse/gespraech_0.htm).

${ }^{5}$ Auch den Videoeinsatz im Informationsportal Gesprächsforschung werden wir im Folgenden nicht weiter behandeln. Videos werden zwar in der zugehörigen „Gesprächsforschung - Online-Zeitschrift zur verbalen Interaktion“ zur Illustration und als Belege verwendet und lassen sich darin - quasi als Anhänge zu den Zeitschriftenartikeln - herunterladen. Sie werden aber nicht hypermedial in einen fortlaufenden Lehr- oder Analysetext eingebettet.

${ }^{6}$ Christa Stocker: „Studien-CD Linguistik. E-Learning für einen breiten Markt.“ Bericht zum Erwerb des Zertifikats in E-Learning an der Universität, ETH und Pädagogischen Hochschule Zürich, 4.2.2005.
} 
- Online-Systeme bieten darüber hinaus Möglichkeiten zur Kommunikation der Nutzer untereinander (insbesondere über Foren) und der Nutzer mit den Autoren in Form von Kommentaren und kritischen Bewertungen; sie können als Feedback zur Verbesserung, Strukturierung und Erweiterung der inhaltlichen Angebote und der Bedienungsoberfläche des Systems genutzt werden.

Die verschiedenen Systeme verfolgen nach Auskunft der Autoren unterschiedliche Zielsetzungen, z.B.:

- EGon ist eine multimediale und internetgestützte Einführung in die Theorie und Praxis der Gesprächsanalyse, die insbesondere Anfängern den Einstieg in die Gesprächsforschung erleichtern soll: „Wir wollen Interesse an der Gesprächsforschung wecken, ihre Grundbegriffe, Konzepte und Verfahren vorstellen und Gelegenheit zur praktischen Umsetzung des Gelernten bieten.“

- Miteinander reden dient der „Entwicklung eines Online-,,Lernpäckchens“ zum Thema Gesprächsanalyse. Dabei sollen mithilfe der fertiggestellten Lernsoftware ausgewählte historische Aspekte, Kernbegriffe und -theorien der linguistischen Gesprächsanalyse vorgestellt werden.

- Die Studien-CD Linguistik versteht sich als „elektronisches Lehrmittel mit über 80 Lerneinheiten zu den Kernbereichen der Linguistik“; die CD soll „individuelle Lernwege“ ermöglichen und die „selbständige Aneignung von Wissensinhalten und Analysefertigkeiten“" unterstützen.

\section{Verwendung von Audio- und Videodateien in hypermedialen Systemen zur Gesprächsanalyse}

Im Folgenden werden wir anhand unserer Auswertungen der verschiedenen hypermedialen Angebote über die Verwendung von Audio- bzw. Videodaten informieren und versuchen, den Status von Mediendateien im gesprächsanalytischen Arbeitsprozess zu bestimmen. Danach werden wir auch Beispiele für Hypermedia in GAIS nennen.

In den 70er bis 90er Jahren war auf Grund technischer Zwänge eine Trennung von Audio- und Videoaufnahme und Transkript bei der Korpusarbeit (insbesondere bei Dokumentation und Selektion analyserelevanter Stellen) und bei der Publikationspraxis in Printmedien gängig. Printmedien erlaubten nur eine Präsentation von Texten, also Transkriptausschnitten zu Analysen und ganzen Transkripten in Materialbänden. Der Zugriff auf Gesprächsaufnahmen war schon bei der gesprächsanalytischen Arbeit, vor allem für die kontrollierende Rezeption gesprächsanalytischer Arbeiten aber nur mit besonderem Aufwand möglich, etwa auf gesonderte Bestellung.

Die in den neuen technischen Systemen mögliche unmittelbare Verknüpfung von Gesprächsaufnahme und darauf bezogenen Texten, also Transkripten und Analysen, lässt sich nach MacWhinney auch als „Konnektivität" („,connectivity“) bezeichnen. Sie ist in einer Abfolge von fünf historischen Perioden der Datensammlung die ausgebauteste Stufe; historisch vorangegangen sind 
- impressionistische Beobachtungen mit naiven Spekulationen,

- $\quad$ selektive Tagebuchstudien zur empirischen Erfassung von Spracherwerb ${ }^{7}$,

- Transkripte von Tonband oder Video, die erstmals quantitative Analysen ermöglichten,

- Computerunterstützung beim Transkribieren, zur Vereinfachung der Reproduzierbarkeit, zur automatischen oder teilautomatischen Analyse, wodurch größere Datenmengen verarbeitet werden können und Kooperationen möglich werden. ${ }^{8}$

„Konnektivität“ ist zum Einen als Datenaustausch über Internet, E-Mail usw. zwischen Computern zu verstehen. Die andere Lesart von „Konnektivität“ betrifft die zwischen Daten als Integration verschiedener Medien - Text, Bild, Ton, Video, also das, was für gesprächsanalytische Systeme Hypermedialität ausmacht. Diese Konnektivität bedeutet mehr Unterstützung beim Transkribieren durch synoptische Präsentation und Navigation in einer gemeinsamen Benutzeroberfläche und führt zu einer Aufhebung der scharfen Trennung zwischen Aufnahme und Transkript. ${ }^{9}$

Die Zusammenführung erfolgt aber relativ zu Forschungs- und Darstellungsinteressen, denn nicht in jedem Fall ist die volle Präsentation (d.h. Video und Transkript) angezeigt, eine selektive Präsentation (z.B. nur Transkript, nur Audio, Audio mit Transkript) muss nicht Indiz technischer Beschränktheit oder Rückständigkeit, sondern kann durch unterschiedliche Fokussierungsmöglichkeiten motiviert sein. ${ }^{10}$

Online-Systeme stellen für die analytische Arbeit und die Darstellung der Ergebnisse eine synoptische oder sogar synchrone Präsentation zur Verfügung, bei der Audio- oder Videodateien mit Transkriptsegmenten verlinkt sind. Der Einsatz von Videodateien in

7 Von MacWhinney (2006, S. 7) als „Baby Biographies“, d.h. Sprachentwicklungsstudien, beschrieben.

${ }^{8}$ MacWhinney (2006, S. 9) beschreibt „,connectivity“ als Aufhebung der scharfen Grenzen zwischen Text, Ton und Bild: „This new hardware has now opened up the possibility for multimedia access to digitized audio and video from links inside the written transcripts. In effect, a transcript is now the starting point for a new exploratory reality in which the whole interaction is accessible from the transcript."

9 Vgl. ein Vortragshandout des EXMARaLDA-Entwicklers Thomas Schmidt unter http://www1.unihamburg.de/exmaralda/Daten/4D-Literatur/Vortraege-Dokumente/Handout SFB 26092002.pdf

${ }^{10}$ Auf der 6. Arbeitstagung „Neuere Entwicklungen in der Gesprächsforschung“ in Freiburg vom 29.-31. März 2000 wurde ein Workshop „Transkript, Audio, Video: Bestimmt das Aufzeichnungsmedium das Ergebnis?" in Form kontrastiver Datensitzungen veranstaltet. Anhand unterschiedlicher Datenbasen Transkript, Audioaufzeichnung, Videoaufzeichnung und Videoaufzeichnung plus Transkript - erarbeiteten die Teilnehmer der Gruppensitzungen deshalb die jeweiligen Vor- und Nachteile der Aufzeichnungsart für die gesprächsanalytische Auswertung. In den Ergebnissen der Gruppe, der lediglich das Transkript zur Verfügung stand, wurden die Stärken der Transkriptanalyse deutlich: die Erfassung sequenzieller Ordnungsstrukturen, die detailliert erfasste Datengrundlage, die Deutungsvielfalt des Transkripts. Audio- und vor allem Videoaufnahmen bieten Vorteile, wenn es neben der Erhebung verbaler Daten auch auf die Berücksichtigung paraverbaler und nonverbaler Zeichen ankommt und das Ereignis um die Kommunikation herum mit erfasst werden muss. Das Mehr an Daten wird aber mit größerer Komplexität und - insbesondere bei Fernsehaufzeichnungen - der Selektivität der Kameraperspektive erkauft. Letztlich kann wohl nur eine Methodenkombination allen Anforderungen gerecht werden. Das Ziel des Workshops war, ein tieferes Bewusstsein für die Reichweite, aber auch die Begrenztheit der jeweiligen Aufzeichnungsmedien zu schaffen (vgl. Klemm/Leister/Schulz-Nötzold 2000, S. 36). Zur Methodologie gesprächsanalytischer Arbeitsschritte von einer vorsequenziellen Auswertung über Sequenzanalyse, Konzeptualisierung/Theoretisierung hin zur Ergebnispräsentation relativ zu einem vorhandenen Korpus von Videoaufnahmen vgl. auch Schmitt (2001). 
gesprächsanalytischen Informationssystemen lässt sich in Anlehnung an die genannte historische Entwicklung funktional bestimmen. Der nachfolgende Überblick über die Verwendungsmöglichkeiten ist als Steigerung von illustrativen über didaktische bis hin zu analytischen Funktionen gegliedert.

\subsection{Einfache Illustration}

In Miteinander reden wird im Modul „Gesprächsforschung“ auf Seite 4 ein Beispiel zur Demonstration der Verbindung von Transkript und Ton gegeben.
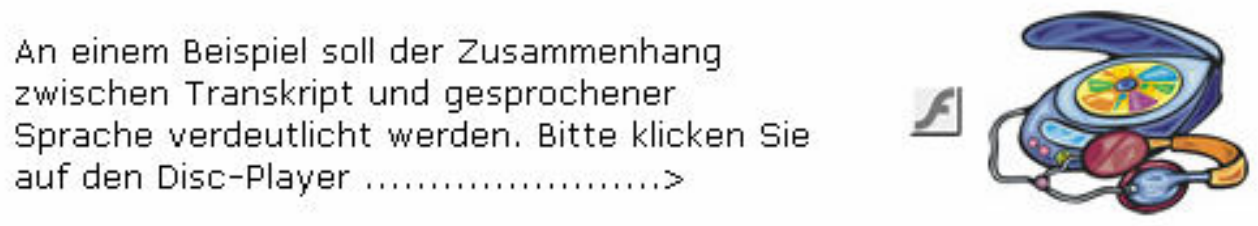

Abbildung 1: Arbeitsanweisung aus Miteinander reden

Das Transkript wird dabei segmentweise eingeblendet, auf diese Weise lassen sich gut die Transkriptgenauigkeit überprüfen und die prosodischen Annotationen sinnfällig machen. Über die illustrative Funktion hinaus wird das Potenzial einer Verknüpfung mit Analysen aber nicht weiter ausgeschöpft, es handelt sich lediglich um eine Bebilderung von Textangeboten unter Verzicht auf Reflexion.

\subsection{Instruktive Illustration}

In der Studien-CD Linguistik findet sich unter „Gesprächsanalyse“/,Methodik“/,Umgang mit Gesprächen“ ein Videobeispiel, das dazu dient, die Flüchtigkeit von Gesprächen zu belegen, wenn kein Transkript oder andere Formen der Ereignissicherung zur Verfügung stehen. Der Rezipient wird dabei auf die konstitutiven Bedingungen einer Interaktionsrezeption hingewiesen. Dazu wird ein Video fast ganz ohne Navigationsmöglichkeiten angeboten: Bei Aufruf der Seite startet das Video automatisch, die Wiedergabe pausiert bei Klick auf das Video und wird bei erneutem Klick fortgesetzt. Der Verzicht auf weitere Navigationsmöglichkeiten an dieser Stelle korrespondiert jedoch mit dem angestrebten didaktischen Zweck: Gezeigt werden soll, dass wegen der Flüchtigkeit der Gespräche eine nur auf Erinnerung basierende Beschäftigung mit ihnen wissenschaftlich fruchtlos und eine reine Videoaufnahme gesprächsanalytisch nutzlos ist, wenn die Gespräche nicht durch eine sog. „Datenerhebung“ dokumentiert werden.

Das nachfolgende Bildschirmfoto zeigt, wie das Konzept „Datenerhebung“ arbeitspraktisch in Handlungsmaximen (,Faustregeln“) umzusetzen ist. Sie betreffen die Gesprächsaufzeichnung und die Verwaltung von Metadaten. Außerdem sieht man links die Schlichtform eines Videoplayers, der hier ganz auf Navigationsschaltflächen verzichtet: 


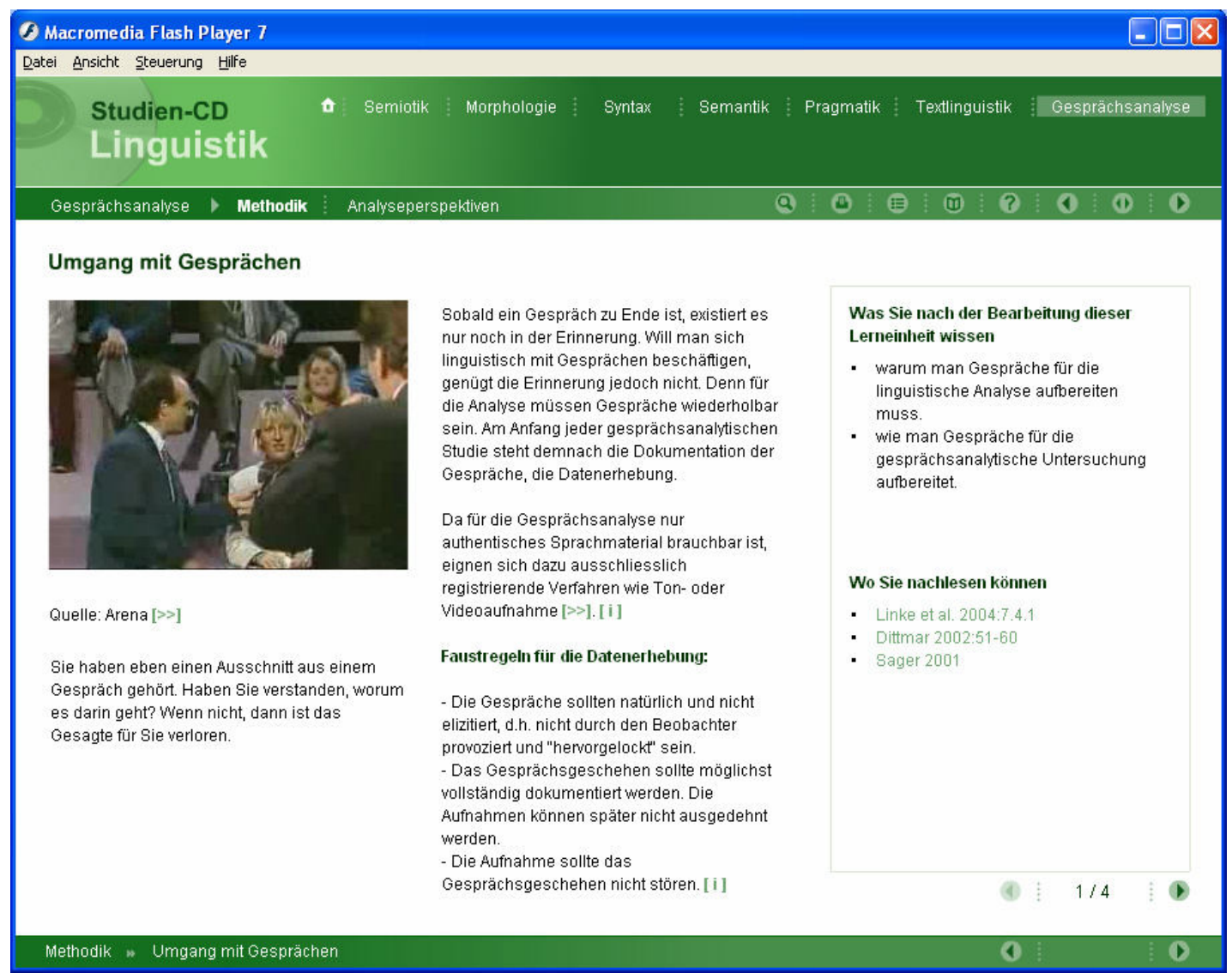

Abbildung 2: Studien-CD Linguistik, Seite aus dem Bereich „Gesprächsanalyse“ $\rightarrow$ „Methodik“ $\rightarrow$ „Umgang mit Gesprächen“

\subsection{Didaktische Demonstration}

Die Studien-CD Linguistik demonstriert dann an anderer Stelle (s. ,Gesprächsanalyse/Methodik/Medienwahl/Medienwahl: Audio vs. Video") auf vier Seiten eine didaktische Einführung in verschiedene Präsentationsweisen von Gesprächsmaterialien. Im Zentrum steht der Vergleich von Rezeptionseigenschaften der Materialformen Audio und Video.

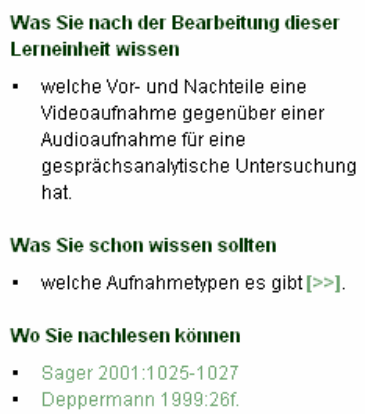

Abbildung 3: Studien-CD Linguistik, Seite aus dem Bereich „Gesprächsanalyse“ $\rightarrow$ „Methodik“ $\rightarrow$ "Medienwahl“ $\rightarrow$,Medienwahl: Audio vs. Video“(S. 1, Ausschnitt) 
Auf dieser ersten von den vier Seiten wird die didaktische Zielsetzung der Studien-CD deutlich gemacht: Explizit werden die Voraussetzungen genannt, die für eine erfolgreiche Rezeption notwendig sind (mit einem CD-internen Link, um die Beschäftigung mit notwendigen Wissensvoraussetzungen nachzuholen; dies geschieht u.a. vorsorglich für den Fall, dass der Nutzer hypermedial zuerst auf die aktuelle Seite gelangt ist und darum noch nicht weiß, ,welche Aufnahmetypen es gibt"). Dazu tritt eine vorgreifende Verdeutlichung, welches Ergebnis diese Lerneinheit bringen soll. Interessant dabei ist, dass auch Nachteile einer Videoaufnahme gegenüber einer Audioaufnahme dargestellt werden. ${ }^{11}$ Es folgt eine explizite Arbeitsanweisung, was man auf dieser und den nachfolgenden Seiten tun soll.

Der Ausschnitt aus dem Fernsehgespräch wird hier als Audio- und als Videodatei über zwei Player mit ähnlicher Navigations-Oberfläche präsentiert. Die Steuerungsleiste wird an anderer Stelle (im Hilfe-Menü) erklärt. Die elementaren Navigationsmöglichkeiten ermöglichen eine wiederholte Rezeption der Mediendatei.

Auf der zweiten Seite wird die Audioaufnahme durch ein Transkript angereichert, und es werden die analytischen Möglichkeiten dieser Kombination kommentiert: die Fokussierung der Orientierung des Gesprächsanalytikers auf die verbale und paraverbale Ebene des Gesprächsgeschehens unter Ausblendung des Nonverbalen. Dies wird hier bewertungsneutral dargestellt, lässt sich freilich auch als konstruiertes Manko interpretieren. Als Nutzen oder ökonomischer Effekt steht dem aber eine Reduktion des Transkriptionsaufwands gegenüber.

Auf der dritten Seite wird der Mehrwert der Videoaufnahme, auch Informationen zum nonverbalen Verhalten zu liefern, durch ein entsprechend angereichertes Transkript belegt. Daneben wird hier auch noch der praktische Nutzen der Videoaufnahme beim Transkribieren betont.

Auf der abschließenden vierten Seite schließlich werden Audio- und Videoaufnahme in Bezug auf ihr analytisches Potenzial und ihre Implikationen für den Transkriptionsaufwand und die Veränderung der Aufnahmesituation diskutiert. Als Fazit gibt es jedoch nur einen sehr allgemein gehaltenen methodischen Hinweis, dass das Medium aus forschungsökonomischen Gründen zu der zu bearbeitenden gesprächsanalytischen Fragestellung passen müsse:

\footnotetext{
${ }^{11}$ Genannt wird, (a) dass Datenvolumen, Informationsdichte und damit auch Transkriptionsaufwand bei Videoaufnahmen größer als bei Audioaufnahmen seien (S. 4); (b) dass aber auch eine Videoaufnahme eine Informationsreduktion bedeute, weil der Gesprächsausschnitt vollständig durch die Kameraeinstellung(en) festgelegt werde, was besonders bei Fernsehaufnahmen deutlich werde (S. 3); (c) dass Videoaufnahmen stärker in die Gesprächssituation eingriffen, das Gesprächsgeschehen verändern und damit die Authentizität der Sprachdaten vermindern könnten (S. 4). Wichtig wäre ein zusätzlicher kritischer Hinweis, dass die Beschränkung der Audioaufnahme auf eine Dokumentation des verbalen und paraverbalen Gesprächsgeschehens nicht notwendig ein funktionales Manko gegenüber dem Video ist. Die Benutzung von Audioaufnahmen (obwohl auch ein Video zur Verfügung steht) kann dazu dienen, die Aufmerksamkeit bei der didaktischen Rezeption oder bei der Analyse auf andere Aspekte wie z.B. die prosodische Gestaltung zu lenken, und damit funktional sinnvoll sein.
} 


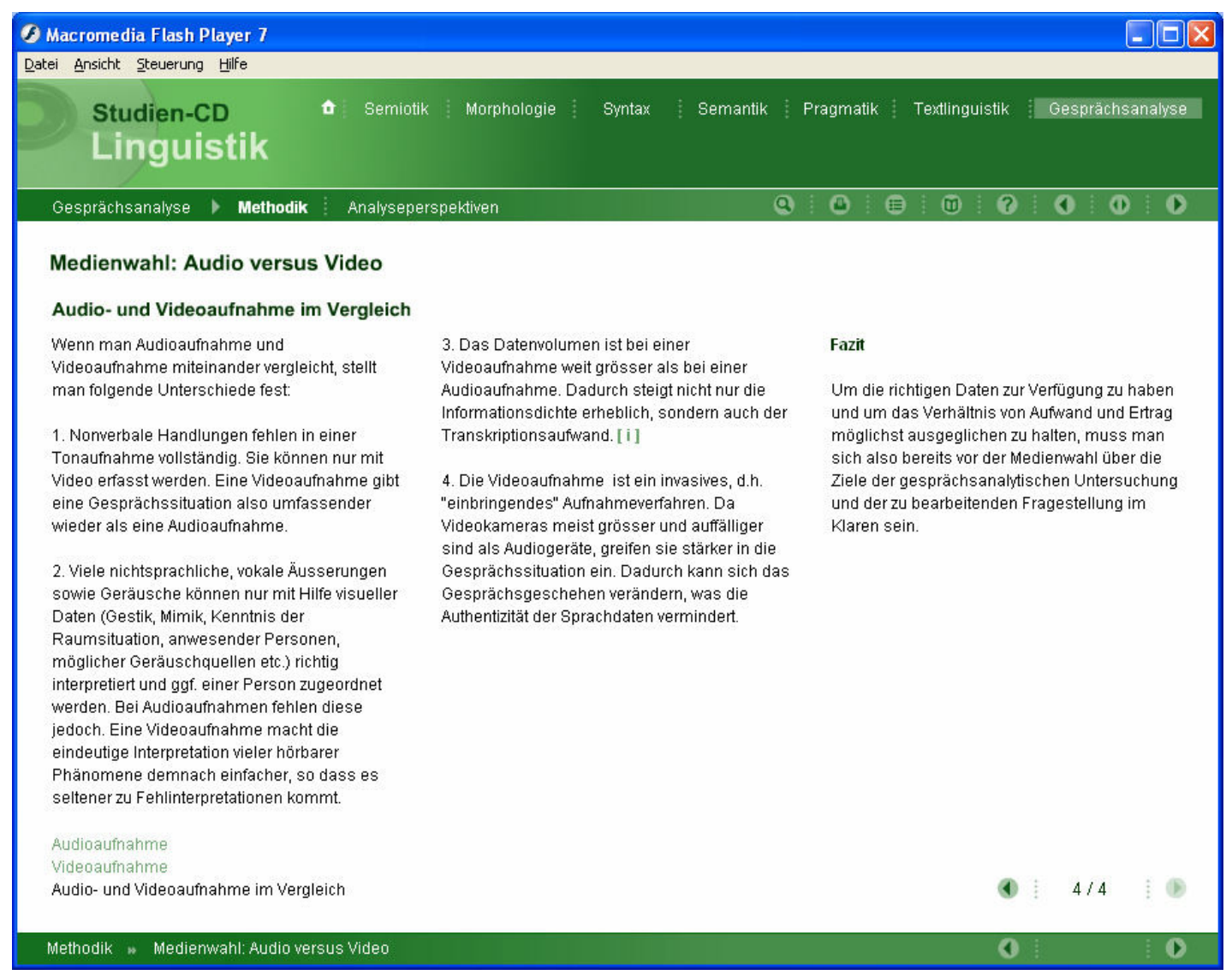

Abbildung 4: Studien-CD Linguistik, Seite aus dem Bereich „Gesprächsanalyse“ $\rightarrow$ „Methodik“ $\rightarrow$ „Medienwahl“ $\rightarrow$ „Medienwahl: Audio vs. Video“ (S. 4)

\subsection{Analysepraktische Zwecke}

Videos können Übungsaufgaben aber nicht nur zu illustrativen oder didaktischen Zwecken beigegeben werden. Sie dienen auch dazu, Analysen zu erleichtern oder überhaupt erst handwerkliche Voraussetzungen für Analysen zu schaffen - oder um im System angebotene Musteranalysen für Nutzer nachvollziehbar zu machen. Das kann in einer Erleichterung bei der Sprecheridentifizierung und einer Zuordnung von Redebeiträgen bestehen, es können aber auch andere Hilfen bei Transkription und Analyse gegeben werden. Dazu dienen Analyseinstrumente wie Standbilder, Einzelbildschaltung, Schleifen, Zeitlupe. 

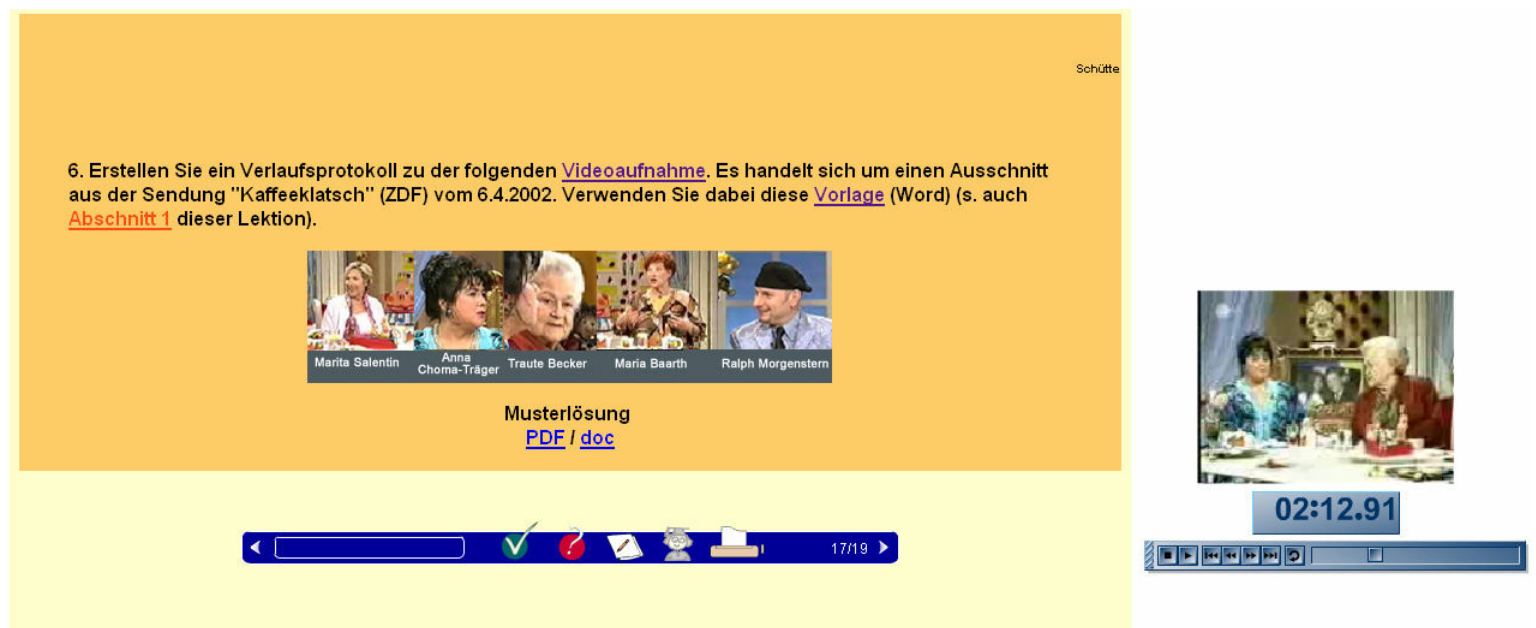

Abbildung 5: EGon: aus einer Lektion zur Methodologie der Gesprächsforschung (4.2-17)

In der EGon-Lektion zur Methodologie der Gesprächsforschung (4.2-17) erleichtern das Video und die Vignetten der Gesprächsbeteiligten (jeweils mit Namensnennung, unter der Aufgabenformulierung, s. Abbildung 5) lediglich die Sprecheridentifizierung für die Aufgabe, ein Verlaufsprotokoll des Fernsehgesprächs zu erstellen. Die Musterlösung dafür enthält nur die handlungsschematischen Funktionen der Redebeiträge mit Zuordnung zu Akteuren und Zeitabschnitten sowie Kommentare zur Sprechweise (Dialektalität und Emotionalität des Redebeitrags), z.B. auszugsweise in Tabellenform so:

\begin{tabular}{|l|l|l|l|}
\hline Zeit & Sprecher & Inhalt/Handlung & Kommentar \\
\hline $1: 23: 26$ & Moderator & $\begin{array}{l}\text { Hinführung zum Thema, Eröffnung des Boh- } \\
\text { len-Themas mit Blick auf Estefania, direkte } \\
\text { Frage an Maria }\end{array}$ & $\begin{array}{l}\text { zitiert Bohlen, betont } \\
\text { kölnischer Dialekt }\end{array}$ \\
\hline
\end{tabular}

Multimodale Aspekte (Gestik, Mimik, Proxemik, Sitzordnung usw.) sind für diese Aufgabe, den Kommunikationsverlauf darzustellen, irrelevant.

\subsection{Video zur Verdeutlichung des aktuellen Gesprächs-Settings}

Ein Spezialfall der unter 3.4 genannten Voraussetzungssicherung für Analysen und ihre Nachvollziehbarkeit liegt vor, wenn das Video über einen rein illustrativen Zweck hinaus das Gesprächs-Setting verdeutlicht. Dazu zählen die Sitzordnung, der gemeinsame Wahrnehmungsraum der Gesprächsbeteiligten und der Öffentlichkeitsgrad des Gesprächs. Bei Ausschnitten aus Fernsehgesprächen kann man häufig erkennen, ob das Gespräch vor Studiopublikum oder in einer abgeschlossenen Runde geführt wurde; das hat Implikationen für den interaktiven Vollzug, ob es z.B. für eine Mehrfachadressierung an die unmittelbar Gesprächsbeteiligten und an Zuschauer empirische Korrelate gibt ob etwa Protagonisten durch kurze Blickrichtungswechsel das Studiopublikum zum Komplizen ihrer Auseinandersetzung mit dem Gesprächspartner zu machen versuchen, ob sie sich so der Wirkung ihrer eigenen Äußerungen vergewissern oder die Äußerungen ihrer Gesprächspartner demonstrativ mimisch kommentieren. 
Ein Beispiel aus einer Aufgabe in GAIS zeigt die Studioatmosphäre einer Club-2Diskussion im österreichischen Fernsehen und macht zugleich die Beteiligten durch einen aufbereiteten Screenshot identifizierbar:

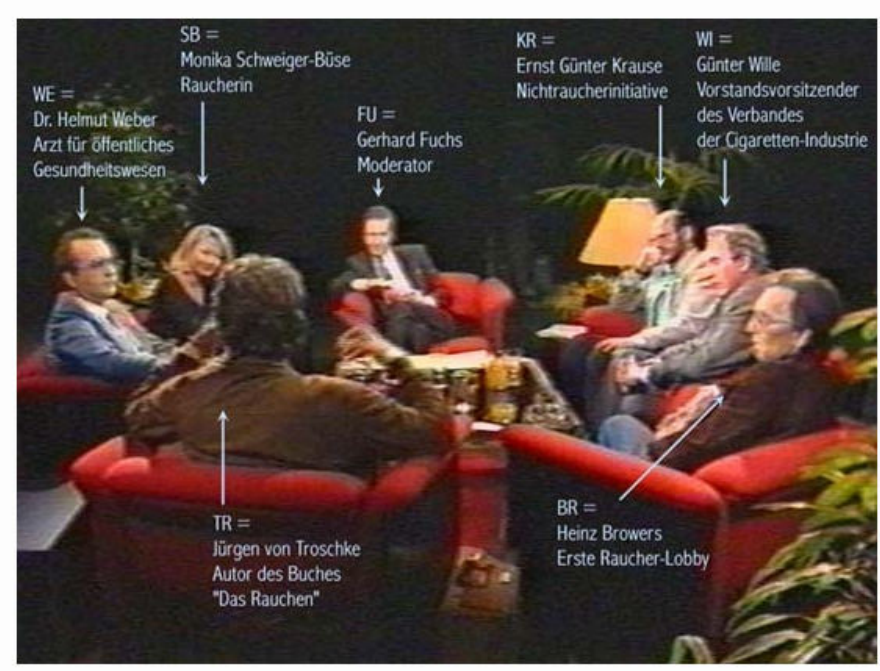

Abbildung 6: Aus einer GAIS-Aufgabe

\subsection{Nonverbales als Analysegegenstand}

Bei der Verwendung von Videos können auch nichtverbale Eigenschaften einer Situation bzw. eines situierten Geschehens zum Gegenstand multimodal orientierter Analysen werden, in denen das Interesse an den nicht-verbalen Anteilen hochgestuft wird. ${ }^{12}$ Das kann Einzelanalysen (zur Demonstration theoretischer Darstellungen) oder Übungsaufgaben betreffen, in denen in der Aufgabenstellung und gegebenenfalls auch in der Musterlösung auf Nichtverbales verwiesen wird, das nur durch Rezeption des Videos kenntlich wird. Wie unterschiedlich diese Möglichkeit genutzt wird, zeigen wir im Folgenden anhand zweier Beispiele.

In der EGon-Lektion zur Gesprächsorganisation ${ }^{13}$ werden multimodale Möglichkeiten in der hier angebotenen Musterlösung nicht ausgeschöpft. Anhand des Videos könnte untersucht werden, welche Rolle der Blickrichtungswechsel des Gastes Katrin Huß beim Sprecherwechsel spielt - ob sie den nächsten Sprecher auswählt oder auf die Selbstwahl des nächsten Sprechers entsprechend reagiert:

\footnotetext{
${ }^{12}$ Zum Konzept multimodaler Interaktion und den in der audiovisuellen Qualität der empirischen Daten begründeten Veränderungen der Interaktionsanalysen vgl. Schmitt (in diesem Band) sowie Schmitt (2004, 2005, i.Dr., i.V.).

${ }^{13}$ Insbesondere zum Sprecherwechsel; 7.4-15, Übung 5, Analyse 2.
} 

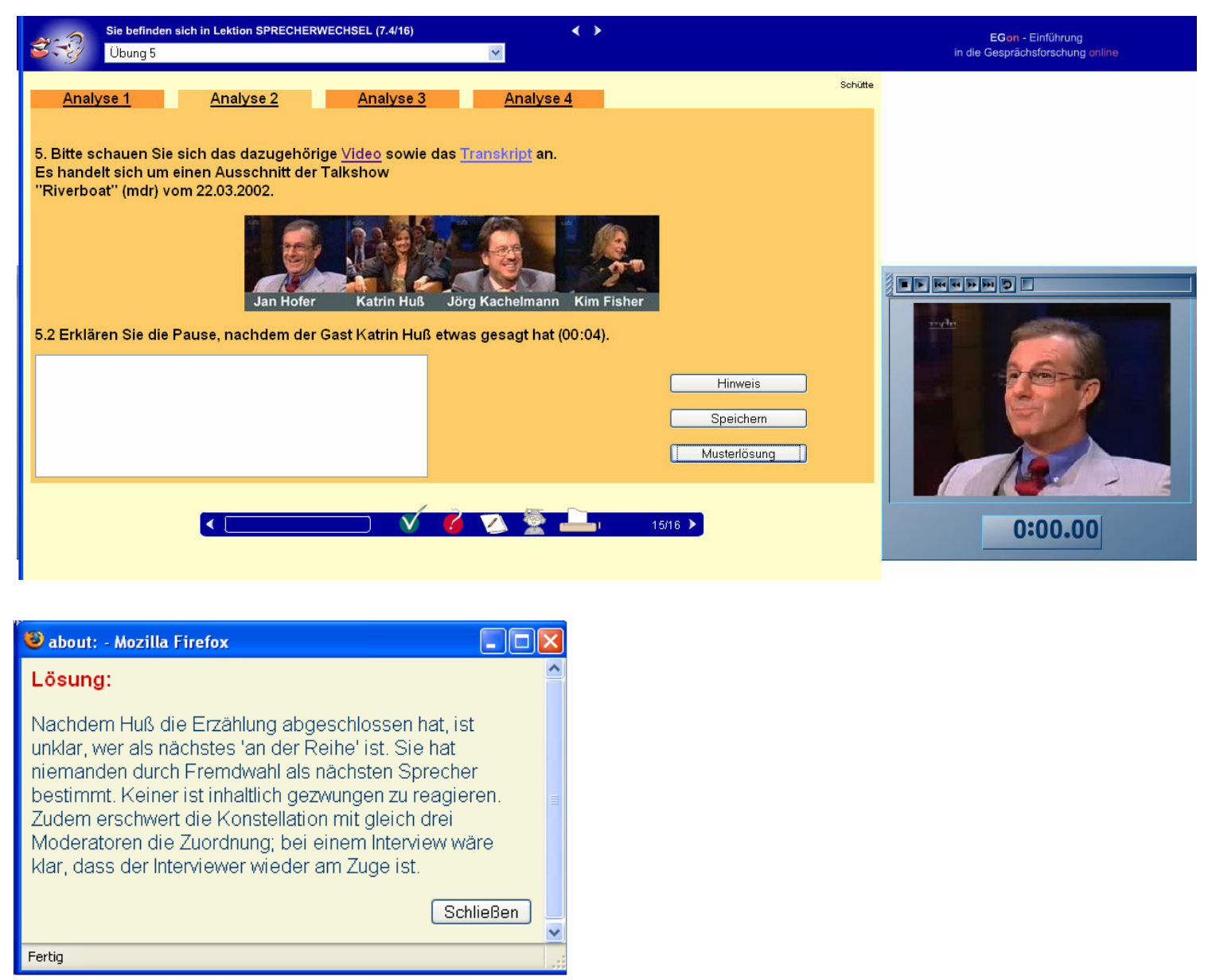

Abbildung 7 und 8: Aus der EGon-Lektion zur Gesprächsorganisation (Sprecherwechsel; 7.4-15, Übung 5, Analyse 2) und Musterlösung

Interessanter ist die zweiteilige Musterlösung bei der nachfolgenden Analyse 3 in EGon: Deren zweiter Teil bezieht sich auf die prosodische (Lautstärke, Sprechgeschwindigkeit) und vor allem nonverbale Komponente (Körperhaltung und Berührung eines Gesprächspartners) bei der Organisation des Sprecherwechsels.

23 about: - Mozilla Firefox
Lösung:
Noch während Kachelmann am Reden ist, beginnt Huß
ihren Beitrag, setzt aber nochmals danach neu an,
nachdem sie Kachelmanns Anspielung mit einem
knappen ja genau abgeschlossen hat. Sie wird dabei
ein bisschen lauter, redet aber vor allem in hohem
Tempo, ohne TRPs zuzulassen. Durch ihre Zuwendung
zu Höfer und das kurze und leichte Berühren
unterstreicht sie, dass sie am Rederecht bleiben
möchte, bis sie die Pointe gesetzt hat.
Fertig

Abbildung 9: Aus der EGon-Lektion zur Gesprächsorganisation (Sprecherwechsel; 7.4-15, Übung 5, Analyse 3) und Musterlösung 
Der Flash-Player in EGon lässt sich vielfältig nutzen - wegen der integrierten Einzelbildschaltung sowie der Möglichkeit, das Video mit dem Slider an einer bestimmten Stelle anzuhalten und von dort ab das Video weiter abspielen zu können. Die Verbindung von Video und Transkript ist dabei allerdings noch statisch: Das Transkript erscheint in einem neuen Fenster im PDF-Format, es ist offensichtlich zum Ausdrucken und Mitlesen während der Video-Rezeption gedacht.

Die bislang präsentierten Beispiele operieren mit sukzessive aufzurufenden Elementen eines Hypertextes, z.B. über Links, die zu einer ausgelagerten Musterlösung führen. Einen qualitativen Sprung ergibt eine synoptische und sogar synchronisierte Präsentation von Mediendatei (Audio oder Video) mit Texten und grafischen Darstellungen zur Transkription, Interpretation und kategorial gebundenen Analyse. Als Beleg für diese besonders starke Integration dient das folgende Beispiel aus eHype.

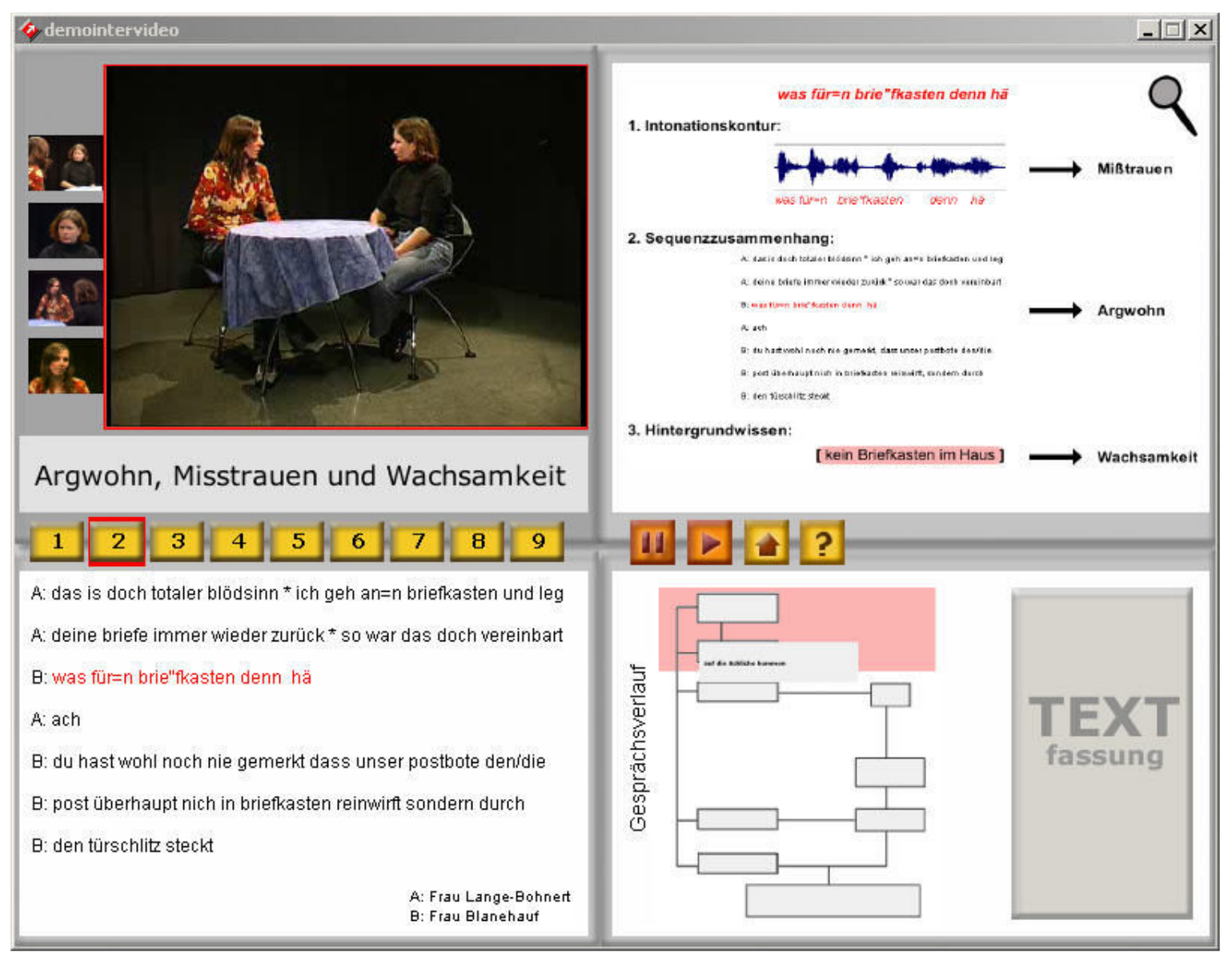

Abbildung 10: eHype, Kursmodul „Der evolutive Sprung“

In der Mitte des Screenshots sieht man einen Balken zur Orientierung darüber, in welcher Etappe der Lernumgebung man sich befindet, und mit Navigationsbuttons zum Video. In den vier Feldern befinden sich links oben das Video, darunter das Transkript, rechts unten das Diagramm zum Gesprächsverlauf und schließlich rechts oben Darstel- 
lungen zur prosodischen Konturierung, zum handlungsschematischen Sequenzzusammenhang und zum Hintergrundwissen, die auf die drei alltagssprachlichen Konzepte „Argwohn“, „Misstrauen“ und „Wachsamkeit“" bezogen werden.

Dies ist ein Beispiel für „Konnektivität“ im Sinne von MacWhinney als Integration verschiedener Medien wie Text, Bild, Ton, Video - so kann Hypermedialität für gesprächsanalytische Systeme forschungspraktisch und didaktisch genutzt werden. Die Konnektivität unterstützt das Transkribieren, das Analysieren und die Rezeption von Analysen durch synoptische Präsentation und Navigation in einer gemeinsamen Benutzeroberfläche und führt zu einer Aufhebung der scharfen Trennung zwischen Aufnahme und Transkript.

In methodischen Beiträgen zur Gesprächsanalyse, zuletzt auch auf dem IDS-Kolloquium „Symbolische Interaktionen“ am 10./11.2.2006, wurden in Vorträgen und Diskussionsbeiträgen die Lesbarkeit von Transkripten und ihre Verabsolutierung zur alleinigen Quelle für gesprächsanalytisches Arbeiten kritisch bewertet. Die synoptische oder sogar synchrone Präsentation von Gesprächsaufnahmen als Audio oder Video, von Transkripten, Analysen und ihren grafisch aufbereiteten Korrelaten ist eine Antwort auf diese Kritik. Freilich kann und will auch eine hypermediale Gesprächsanalyse andere Einwände nicht aushebeln: Der komfortable nichtsequenzielle Zugriff auf bestimmte Episoden einer Gesprächsaufnahme, die beliebige Reproduzierbarkeit und Variierbarkeit der Rezeption und die Verknüpfung mit vielfältigen Formen gesprächsanalytischer Aufbereitung steht in noch stärkerem Kontrast zur Flüchtigkeit des natürlichen Gesprächsereignisses als der traditionelle Zugriff auf analoge Aufnahmen und getrennt davon auf Transkripte. Die genannten Umgangsweisen sind jedoch unabdingbare Voraussetzung für alle gesprächsanalytischen Arbeitsschritte - von der Segmentierung über die Sequenzanalyse bis hin zur Beschreibung von Mustern und ihren Varianten.

\section{Video- und Audiodateien in GAIS}

Videos werden in GAIS in ähnlicher Weise wie in anderen Systemen eingesetzt: als Material für eine Transkriptionsübung und zur Verdeutlichung nonverbalen Verhaltens der Beteiligten im Hinblick auf Proxemik, Gestik, Mimik. Das Video ist insbesondere dann unverzichtbar, wenn auf diese Aspekte im Transkript und/oder in der Analyse Bezug genommen wird. Auch Vorträge auf linguistischen und gesprächsanalytischen Tagungen werden zukünftig in GAIS präsentiert. ${ }^{14}$

Bei den Audioaufnahmen kann, wenn die Analyse mit auditiv gewonnenen prosodischen Zuschreibungen operiert, zusätzlich die prosodische Qualität durch eine grafische und wortweise gelabelte Darstellung von Waveform, Grundfrequenz und Intensität (Energie) elaboriert werden. Derartige hypermediale Aufbereitungen prosodischer Analysen haben wir in anderen Systemen nicht finden können. Die Aufbereitungen dienen der Verifizie-

${ }^{14}$ Der Vortrag auf dem Kolloquium „Symbolische Interaktionen“ zu Ehren von Werner Kallmeyer am 11.2.2006, der diesem Aufsatz zugrunde liegt, wurde zu Test- und Demonstrationszwecken als erster in GAIS eingestellt (vgl. Community $->$ Veranstaltungen -> Vorträge -> GA hypermedial, http://gais.idsmannheim.de/community/veranstaltungen/vortraege/ga_hypermedial/). Das Vortragsmanuskript ist ebenfalls als Download verfügbar. 
rung interpretativer und auditiver Konzepte und als Anregungen für den Nutzer, bei späteren eigenen Analysen sich selbst solcher apparativer Möglichkeiten zu bedienen. Die Grafik wird dann mit dem Ausschnitt aus dem Sprachsignal und einer „summenden“ Resynthese der Grundfrequenz verlinkt, um die Grundfrequenzanalyse sinnfällig zu machen wie in der folgenden Abbildung zu sehen ist. ${ }^{15}$
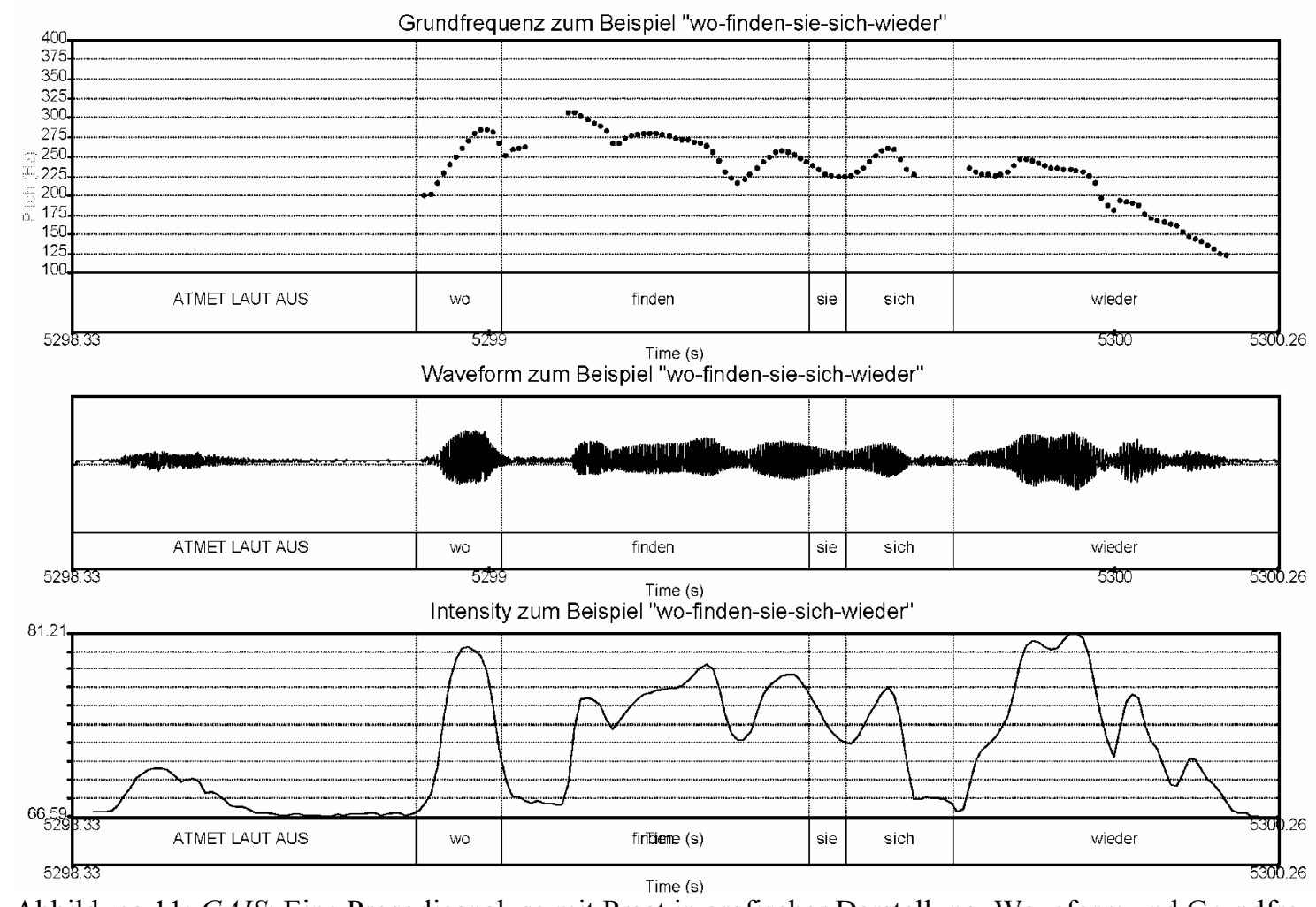

Abbildung 11: GAIS: Eine Prosodieanalyse mit Praat in grafischer Darstellung. Waveform und Grundfrequenzanalyse sind dabei mit Ausschnitten aus dem Sprachsignal verknüpft.

Als letztes Beispiel möchten wir eine Entwicklungsperspektive für GAIS in Form einer stärkeren Verknüpfung zwischen Video und verschiedenen Texten ${ }^{16}$ aufzeigen: Die Rezeption von Text und Video über eine synoptische Präsentation hinaus kann durch eine Synchronisation erleichtert werden. Dabei wird das aktuelle Transkriptsegment durch einen Cursor oder durch farbliche Hinterlegung markiert, so dass der Nutzer den aktuellen Abschnitt des Transkripts dem Video besser zuordnen kann. Eine besonders elegante Lösung dazu ist das in Nijmegen am MPI für Psycholinguistik entwickelte ELAN ${ }^{17}$, das bislang leider nur offline als Transkriptions- und Annotationswerkzeug und als Player verwendet werden kann. ${ }^{18}$ Hier ein Beispiel für ein Transkript, das in EXMARaLDA ${ }^{19}$

${ }^{15}$ Diese prosodischen Analysen werden mit Praat erstellt (s. http://www.praat.org).

${ }^{16}$ Wie es ansatzweise auch eHype vorführt; s. Abschnitt 3.7

${ }^{17} \mathrm{http}: / /$ www.mpi.nl/tools/elan.html.

${ }^{18}$ Eine Web-Anwendung unter dem Namen ANNEX (,Annotation Exploration tool in the MPI web-based framework for archive exploration and enrichement") ist aber kurz vor der Veröffentlichung (vgl. http://www.mpi.nl/annex/, wo auch Screenshots für unterschiedliche Betrachtungsmodi angeboten werden). 
erstellt und nach ELAN exportiert wurde - ein Ausschnitt aus einer Talkshow der Reihe „Boulevard Bio“, hier eine Szene mit dem Talkgast Thomas Gottschalk, der inszenatorisch vorführt, wie einer seiner eigenen Late-Night-Show-Gäste bei anderer Gelegenheit diese Late-Night-Show karikiert:

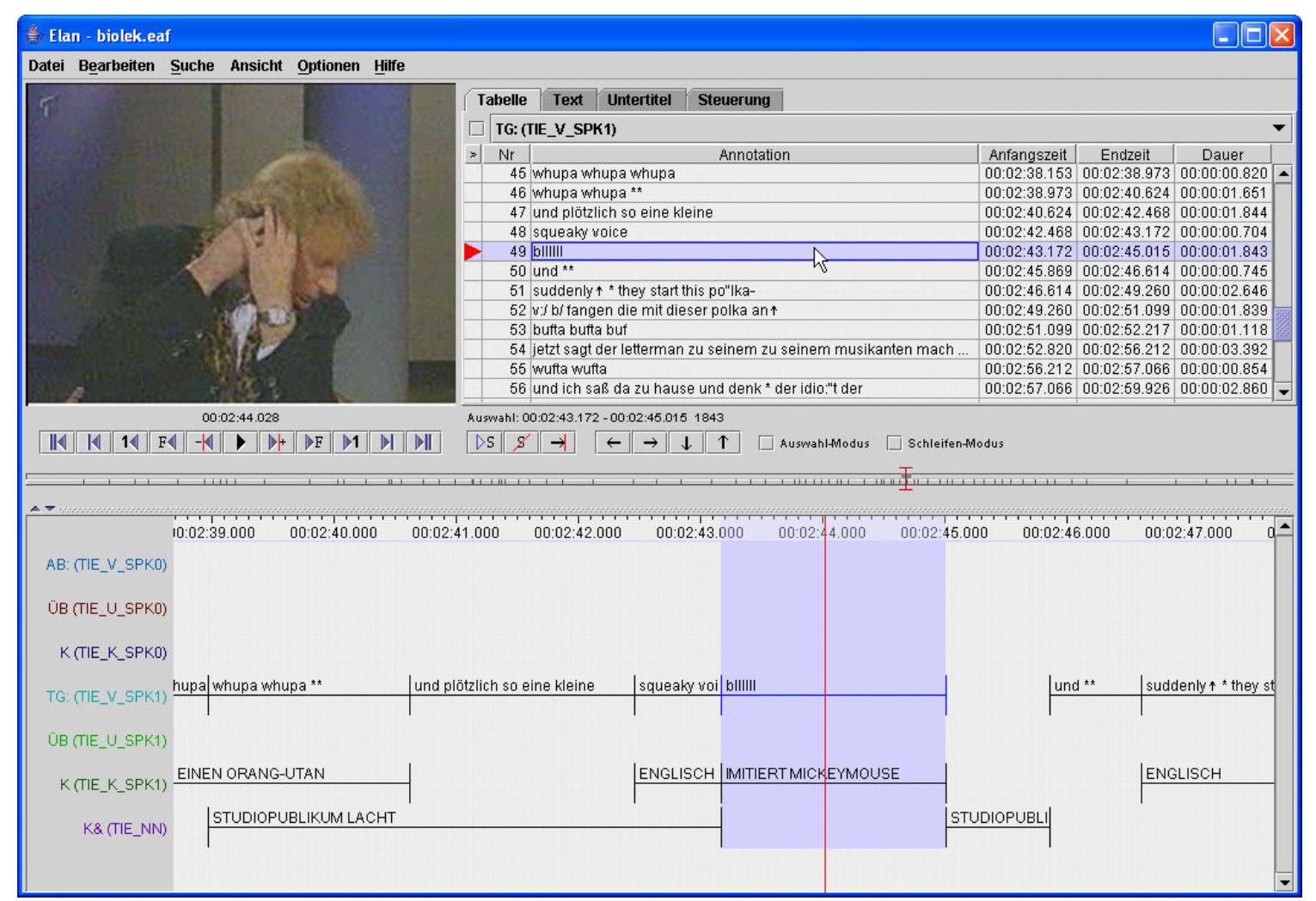

Abbildung 12: ELAN, synchrone Präsentation von Video und Transkript-Annotationen

\section{Ausblick}

Die gesprächsanalytische Arbeitsweise hat im Lauf ihrer noch recht kurzen Entwicklung v.a. durch die technologischen Neuerungen Impulse erfahren. Das galt für die Möglichkeiten der Datenaufzeichnung mit der Verkleinerung der Aufnahmegeräte und der damit verbundenen geringeren Auffälligkeit in alltagsweltlichen Interaktionssituationen, für die Entwicklung von Videokameras bis zur heutigen Digitaltechnik wie auch für die Erfassung und Verarbeitung von Gesprächsdaten und Gesprächs-Metadaten in Rechnersystemen. Die Möglichkeiten moderner multimedialer und internetbasierter Informationssysteme erweitern die gesprächsanalytische Arbeitsweise nun um ein Vielfaches: Sie reichen hinsichtlich des Umgangs mit Audio- und Videodaten von der bloßen Illustrati-

${ }^{19}$ EXMARaLDA („Extensible Markup Language for Discourse Annotation“) ist ein System von Konzepten, Datenformaten und Werkzeugen für die computergestützte Transkription und Annotation gesprochener Sprache, vgl. http://www1.uni-hamburg.de/exmaralda/. 
on über die didaktisch motivierte Instruktion und die didaktische Demonstration, die Erweiterung und Verbesserung der Analysepraxis bei der Transkription und der Handhabung der Daten bis hin zu unmittelbar analytischen Zwecken der multimodalen Analyse und der integrierten Ergebnispräsentation mit Video, Transkript und Analysen.

Idealtypisch orientiert sich der Einsatz von Videos in Hypermediasystemen an zwei diametral unterschiedlichen Ansprüchen:

- Simulation der Flüchtigkeit des originalen Kommunikationsereignisses und damit eine Nachbildung der natürlichen Rezeptionsbedingungen - zugleich als didaktische Anregung, sich in Bedingungen der Produktion und Rezeption von Gesprächen hineinzuversetzen;

- Video als spezifischer Zugriff auf das natürliche Gesprächsereignis, der durch Reduktion und Anreicherung gekennzeichnet ist - Reduktion der Komplexität des natürlichen Gesprächsereignisses durch Kameraführung und Bildauflösung: Selektion von Betrachtungsperspektiven, dadurch Fokussierung auf bestimmte Beteiligte, bei Totalen Verzicht auf Details, dagegen Anreicherung durch beliebige Reproduzierbarkeit des Abspielens und Manipulationen des zeitlichen Ablaufs; dadurch werden Äußerungen verständlich und Muster deutlich - das sind Zugriffsmöglichkeiten, die den Beteiligten in der originalen Interaktionssituation so nicht zur Verfügung gestanden haben.

Der Einsatz von digitalisierten Videos ist in mindestens vier Phasen des gesprächsanalytischen Arbeitsprozesses ${ }^{20}$ möglich und sinnvoll - die Navigationsmöglichkeiten der Player müssen dabei dem Einsatzzweck angemessen sein:

a) Transkription: Ein in den Transkriptionseditor integrierter oder zugeschalteter Videoplayer dient der Sprecheridentifizierung sowie der Annotation von Gestik, Mimik und Proxemik.

b) Einzelfallanalyse: Ein Videoplayer, möglichst mit vielfältigen Navigationsmöglichkeiten (z.B. Standbild, Einzelbildschaltung, Zeitlupe, Zeitraffer, Schleifen) dient der Analyse des nonverbalen Verhaltens. Die Navigationsmöglichkeiten erlauben Feinanalysen des nonverbalen Verhaltens, auch zur Synchronisierung des verbalen mit dem nonverbalen Verhalten oder zum nonverbalen „Backchannel behaviour“ der gerade nicht aktiven Sprecher.

c) Publikation: Eine hypermediale Publikationsform dient der Veranschaulichung und Überprüfbarkeit von Einzelfallanalysen und Musterbeschreibungen in der Rezeption; sie ist besonders bei komplexen Verhaltensbeschreibungen angezeigt, die nicht oder nur bedingt durch eine entsprechende Annotation im Transkript verständlich und plausibel sind. Das gilt z.B. für Zuschreibungen einer Verhaltensinszenierung.

d) Didaktische Vermittlung, u.a. in der Hochschullehre und in hypermedialen Systemen (offline und online).

Die didaktische Vermittlung gesprächsanalytischer Kompetenzen und Forschungsergebnisse sehen wir nicht als dem gesprächsanalytischen Arbeitsprozess nachgeordnet an. In der Regel wird sie auch nicht arbeitsteilig von Lehrern oder Hochschuldidaktikern be-

\footnotetext{
${ }^{20}$ Umfassender beschreiben wir Veränderungen des gesprächsanalytischen Arbeitsprozesses durch neue Technologien und hypermediale Systeme in Schütte/Spranz-Fogasy (i.Dr.).
} 
trieben, sondern von den AutorInnen selbst. Gerade in der Gesprächsanalyse geht es dabei nicht allein um Musterbeschreibungen zum Forschungsgegenstand, sondern auch um eine Vermittlung der analytischen Mentalität, also der spezifischen gesprächsanalytischen Methodik. Die Handhabung von Videos durch den Benutzer kann dabei den gesprächsanalytischen Erkenntnisprozess nachbilden und so zu einem vertieften Verständnis der gesprächsanalytischen Arbeitsweise verhelfen. Zugleich bietet die Verwendung von hypermedialer Ergebnispräsentation mit Videos in der Hochschullehre, insbesondere im Seminar, dem Autor die Möglichkeit eines Feedback: Welche Beschreibungen sind musterhaft, nachvollziehbar und einleuchtend?

Gesprächsanalyse beschäftigt sich mit einem lebendigen und facettenreichen Gegenstand. Die traditionelle Form, mit linearen Lehrbuchtexten ihre Methode zu vermitteln und Forschungsergebnisse in einer Abfolge von Transkriptausschnitten und darauf bezogenen analytischen Texten zu präsentieren, hat allerdings bislang die Rezeption dieser Forschungsrichtung erschwert. Es ist das Verdienst von Werner Kallmeyer, dass er schon früh das Potenzial eines in den Arbeits- und Vermittlungsprozess integrierten direkten Zugriffs auf die Audio- und insbesondere die Videoaufnahme des Gesprächs erkannt und die Entwicklung hypermedialer Systeme für die Gesprächsanalyse gefördert hat.

\section{Literatur}

Cölfen, Hermann (2006): Bildungsbrücken bauen. Dialogische Entwicklung hypermedialer Propädatika für die Linguistik. Duisburg: Gilles \& Franke.

Cölfen, Hermann/Schütte, Wilfried/Spranz-Fogasy, Thomas (2006): Multimediale Perspektiven in der Gesprächsforschung. Ein Nachbericht zu den Präsentationen von Multimedia-Projekten auf dem 35. Treffen des Arbeitskreises Angewandte Gesprächsforschung (AAG) am 12. - 13. November 2004 in Fulda. In: Gesprächsforschung - Online-Zeitschrift zur verbalen Interaktion 7, S. 35-69. (http://www. gespraechsforschung-ozs.de).

Heidtmann, Daniela (2004): GAIS - Multimediale Wissensvermittlung in der Gesprächsanalyse. In: Schmitz, Ulrich (Hg.): Linguistik lernen im Internet. Das Lehr-/Lernportal PortaLingua. Tübingen: Narr 2004. S. 133-141.

Heidtmann, Daniela/ Schütte, Wilfried/Steiger, Kerstin (2003): GAIS - GesprächsAnalytisches InformationsSystem. Ein hypermediales Lernsystem zur Gesprächsforschung. In: Gesprächsforschung - OnlineZeitschrift zur verbalen Interaktion 3, S. 137-146. (http://www.gespraechsforschung-ozs.de).

Klemm, Michael/Leister, Angela/Schulz-Nötzold, Carsten (2000): Bericht über die 6. Arbeitstagung „Neuere Entwicklungen in der Gesprächsforschung“ in Freiburg vom 29. - 31. März 2000. In: Gesprächsforschung - Online-Zeitschrift zur verbalen Interaktion 1, S. 30-39. (http://www.gespraechsforschung-ozs.de).

MacWhinney, Brian (2006): The CHILDES Project. Tools for Analyzing Talk - Electronic Edition: Volume 1: Transcription Format and Programs Part 1: The CHAT Transcription Format. (http: //childes.psy.cmu.edu/manuals/chat.pdf; letzte gedruckte Version: MacWhinney, Brian (2000): The CHILDES project. Tools for analyzing talk. 3. Aufl. Mahwah, NJ: Lawrence Erlbaum).

Schmitt, Reinhold (2001): Von der Videoaufzeichnung zum Konzept „Interaktives Führungshandeln“: Methodische Probleme einer inhaltlich orientierten Gesprächsanalyse. In: Gesprächsforschung - Online-Zeitschrift zur verbalen Interaktion 2, S. 141-192. (http://www.gespraechsforschung-ozs.de)

Schmitt, Reinhold (2004): Die Gesprächspause: „Verbale Auszeiten“ aus multimodaler Perspektive. In: Deutsche Sprache 32, S. 56-84. 
Schmitt, Reinhold (2005). Zur multimodalen Struktur von turn-taking. In: Gesprächsforschung - Onlinezeitschrift zur verbalen Interaktion 6, S. 17-61. (www.gespraechsforschung-ozs.de).

Schmitt, Reinhold (i.Dr.): Von der Konversationsanalyse zur Analyse multimodaler Interaktion. In: Kämper, Heidrun (Hg.): Sprach-Perspektiven. Germanistische Linguistik und 40 Jahre IDS. Tübingen: Narr.

Schmitt, Reinhold (i.V.) (Hg.): Koordination. Analysen aus multimodaler Perspektive. Tübingen: Narr.

Schmitt, Reinhold (in diesem Band): Videoaufzeichnungen als Grundlage für Interaktionsanalysen.

Schütte, Wilfried (2004): GAIS: Warum Hypermedia für Gesprächsanalyse? In: Schmitz, Ulrich (Hg.): Linguistik lernen im Internet. Das Lehr-/Lernportal PortaLingua. Tübingen: Narr 2004. S. 143-151.

Schütte, Wilfried/Spranz-Fogasy, Thomas (i.Dr.). Gesprächsanalyse - hypermedial, didaktisch und interaktiv. Wie verändern neue Technologien die Arbeitsweise der Gesprächsanalyse? In: Kämper, Heidrun (Hg.): Sprach-Perspektiven. Germanistische Linguistik und 40 Jahre IDS. Tübingen: Narr.

Stocker, Christa/Macher, Daniela/Studler, Rebekka/Bubenhofer, Noah/Crvelin, Daniel/Liniger, Reto/ Volk, Martin (2004): Studien-CD Linguistik - Multimediale Einführungen und interaktive Übungen zur germanistischen Sprachwissenschaft. Universität Zürich und Tübingen: Max Niemeyer Verlag.

Dr. Wilfried Schütte

Prof. Dr. Thomas Spranz-Fogasy

Institut für Deutsche Sprache

Postfach 101621

68016 Mannheim

e-mail: schuette@ids-mannheim.de

spranz@ids-mannheim.de 